\title{
Violência contra escolares no Brasil: Pesquisa Nacional da Saúde do Escolar (PeNSE, 2015)
}

\author{
A nationwide school-based study of violence in Brazil (PeNSE, \\ 2015)
}

Flora Beatriz Proiette Terribele (https://orcid.org/0000-0002-8789-4937) ${ }^{1}$

Tiago Neuenfeld Munhoz (https://orcid.org/0000-0003-1281-9542) ${ }^{2}$

${ }^{1}$ Faculdade de Psicologia, Universidade Federal de Pelotas (UFPel). Av. Duque de Caxias 250, Fragata. 96030-000 Pelotas RS Brasil.f.proiette@gmail.com

${ }^{2}$ Programa de PósGraduação em Epidemiologia, UFPel. Pelotas RS Brasil.

\begin{abstract}
The study aims to describe victimization for domestic violence (DV), knife-related violence $(K V)$, firearm-related violence $(F V)$ and sexual violence $(S V)$. This was a nationwide Brazilian survey with school-based interviews. All 9th grade students from public and private schools in Brazil were invited to participate. The sampling process was at random and cluster-based and performed in three stages: municipalities, schools, and classrooms. Univariate, bivariate and multivariate analyses were carried out considering the sample weights. A total of 100,540 individuals were assessed and the prevalence of victimization for DV, KV, FV and $S V$ was $14.5 \%, 7.9 \%, 5.7 \%$ and $4.0 \%$, respectively. The DV was more frequent in the South-eastern region, FV was more frequent in the Central-Western region and the $K V$ and $S V$ were more frequent in the Northern region. The victimization for $D V$ and $S V$ was higher among the girls, while the FV and $K V$ were greater among the boys. All forms of victimization were more frequent among public school students, among those at age 16 or older, for those whose mothers have a lower educational level and those who have used alcohol or illegal drugs.

Key words Violence, Domestic Violence, Violence against Women, Exposure to Violence, Students
\end{abstract}

Resumo Objetivou-se descrever o perfil de escolares brasileiros acometidos por situações de violência física intrafamiliar (VFI), por objeto perfurocortante (VOC), arma de fogo (VAF) e violência sexual (VS). Trata-se de um estudo transversal de base escolar. Foram avaliados escolares do $9^{\circ}$ ano do ensino fundamental em todo Brasil. A amostragem foi realizada em três estágios: seleção dos municipios, posteriormente das escolas, e por fim as turmas. Análises univariadas, bivariadas $e$ multivariadas foram realizadas considerando-se os pesos amostrais. Foram analisadas informações de 100.540 escolares. Foi observada prevalência de $14,5 \%, 7,9 \%, 5,7 \%$ e 4,0\% para a vitimização por VFI, VOC, VAF e VS, respectivamente. A VFI foi mais frequente na região Sudeste, a VAF foi mais frequente na região Centro-oeste e a VOC e VS foram mais frequentes na região Norte do país. A vitimização por VFI e VS foi maior entre as meninas, enquanto a VAF e VOC foi maior entre os meninos. Todas as formas de vitimização por violência foram mais frequentes em escolares das escolas públicas, entre aqueles com 16 anos ou mais, filhos(a) de mães com nenhuma ou baixa escolaridade e que fizeram uso de álcool ou substâncias ilícitas no mês anterior a entrevista.

Palavra-chave Violência, Violência Doméstica, Violência contra a Mulher, Exposição à Violência, Estudantes 


\section{Introdução}

A violência interpessoal é a forma de violência mais registrada no mundo, sendo definida como uso intencional de força ou poder, de forma real ou ameaçada, contra outra pessoa ou grupo, que cause ou possa causar morte por lesões, danos corporais ou psicológicos e prejuízos no desenvolvimento, tanto por agressões físicas, psicológicas, sexuais, como por negligência ${ }^{1}$. Destaca-se aqui três tipos de violência: a intrafamiliar, quando ocorre entre parentes; a violência por parceiro íntimo, quando o agressor e vítima estão em um relacionamento afetivo; e a violência armada, quando se utiliza armas para ferir ou ameaçar outra pessoa ${ }^{1,2}$.

Em todo mundo, estima-se que $10 \%$ a $15 \%$ de crianças e adolescentes em idade escolar sejam vítimas de algum tipo de violência grave ${ }^{3}$. $\mathrm{Na}$ América Latina, estimativas apontam que uma a cada três crianças e adolescentes foram vítimas de algum tipo de violência no período de doze meses ${ }^{4}$. No Brasil, os adolescentes foram um dos grupos mais vitimizados na última década entre 2009 a $2014^{5}$. O perfil das vítimas pôde ser caracterizado em duas grandes dimensões - as meninas sofreram mais vezes violência intrafamiliar e os meninos foram vítimas mais frequentes de violência armada ${ }^{6}$. Nesse período, as meninas também foram apontadas como as principais vítimas de violência sexual ${ }^{7}$. Ainda, análises de estudos de base escolar, utilizando dados da Pesquisa Nacional da Saúde do Escolar (PeNSE), nas edições de 2009 e 2012, apontaram que, dentre as formas de violência, a física foi a mais frequente para ambos os sexos ${ }^{6,8}$.

Sendo uma violação dos direitos, como previsto no Estatuto da Criança e do Adolescente (ECA), todas as formas de violência devem ser notificadas aos órgãos competentes, como Conselho Tutelar da região da ocorrência ${ }^{9}$. Entretanto, estudos estimaram uma alta taxa de subnotificação pelas instituições responsáveis pela proteção integral à criança e ao adolescente, como as escolas e os serviços de saúde, agravando as situações de vulnerabilidade ${ }^{10,11}$. Além disso, adultos, responsáveis e até as vítimas, podem omitir os episódios de violência, pelo medo das possíveis consequências decorrentes da revelação².

Os episódios de violência podem causar prejuízos à saúde física e mental. A literatura apontou que sofrer violência na adolescência está relacionado ao aumento do risco de aproxima- damente duas vezes de desenvolver transtornos de humor e de ansiedade transtornos por uso de substâncias, como uso abusivo de álcool e drogas ilícitas, na vida adulta ${ }^{12}$. Ainda, os prejuízos não se limitam ao indivíduo, uma vez que a violência ocorre e pode se perpetuar por uma rede complexa de fatores pessoais, de valores culturais de uma sociedade e dos ambientes cotidianos dos quais o indivíduo está inserido ${ }^{13}$.

Sendo um problema de saúde pública, o impacto prejudicial da violência demanda a atenção de diversas instâncias, como os serviços de saúde e assistência social, gerando gastos públicos ${ }^{14}$. Para contribuir com a elaboração de intervenções eficazes que minimizem a ocorrência e os danos desse fenômeno, o objetivo desse estudo é descrever a prevalência de adolescentes expostos às situações de violência intrafamiliar, violência sexual e violência armada e analisar os fatores associados, utilizando dados da PeNSE de 2015.

\section{Metodologia}

\section{Desenho do estudo}

Esta pesquisa analisou os dados da Pesquisa Nacional de Saúde do Escolar (PeNSE) de 2015. A PeNSE é um inquérito epidemiológico de base escolar, realizada pelo Instituto Brasileiro de Geografia e Estatística (IBGE), com iniciativa do Ministério de Saúde e da Educação do Brasil, e realiza o monitoramento da população adolescente brasileira (10 a 19 anos). A coleta de dados foi realizada entre abril e setembro de 2015 , por meio de um questionário estruturado e autoaplicado. O questionário foi elaborado contemplando a metodologia recomendada pela Global School-based Student Health Survey (GSHS), desenvolvida pela Organização Mundial da Saúde (OMS), que orienta as pesquisas epidemiológicas que focam nas causas de morbimortalidade em adolescentes escolares. No ano de 2009, primeira fase da PeN$\mathrm{SE}$, foram realizados pré-testes do questionário em escolares do $9^{\circ}$ ano do ensino fundamental em oito escolas (públicas e privadas) nos Municípios de Mesquita (Rio de Janeiro), Rio de Janeiro (Rio de Janeiro), Belém (Pará), Recife (Pernambuco) e Luziânia (Goiás). Os resultados dos prétestes mostraram boa adequação e compreensão do questionário, mantendo as perguntas nas edições de 2012 e 2015. A pergunta sobre violência sexual foi incluída na edição de 2015. 


\section{Participantes e amostragem}

A amostra foi de 102.072 estudantes respondentes, matriculados no ano letivo de 2015, cursando o nono ano do ensino fundamental, nos turnos diurnos, de escolas públicas e privadas, das zonas urbanas e rurais, das 26 capitais do Brasil e Distrito Federal. A escolha dos alunos do nono ano está relacionada a escolarização mínima necessária para compreensão e resposta ao questionário bem como pela similaridade com a faixa etária avaliada pela pesquisa GSHS conduzida pela OMS que embasou os questionários das edições da PeNSE ${ }^{15}$. A PeNSE utilizou três estágios de seleção da amostra com base no Censo Escolar de 2013. As unidades primárias foram os municípios, as unidades secundárias as escolas, e as terciárias foram as turmas, sendo que as escolas foram selecionadas de acordo com o número de turmas do nono ano e as turmas foram selecionadas de forma aleatória. Todos os alunos, das turmas selecionadas que estavam presentes no dia da coleta, foram convidados a responderem o questionário. Para o cálculo do tamanho amostral, foram utilizados como parâmetros a prevalência de $50 \%$, margem de erro de 3 pontos percentuais e nível de confiança de 95\%.

\section{Variáveis de desfecho}

Os desfechos estudados foram vitimização de violência física intrafamiliar (VFI); vitimização por violência sexual (VS) e violência armada, incluindo envolvimento em brigas com arma de fogo (VAF) e com objeto perfurocortante (VOC). Para avaliar a prevalência de VFI, a PeNSE utilizou a pergunta: "Nos últimos 30 dias, quantas vezes você foi agredido(a) fisicamente por um adulto da sua família?". Foram considerados como vítimas de VFI todos os escolares que relataram terem sofrido uma ou mais agressões nos últimos 30 dias. Para avaliação da VS, perguntou-se: "Alguma vez na vida você foi forçado(a) a ter relação sexual?". Todos que responderam "sim" para essa pergunta foram consideradas vítimas de VS.

Já sobre a ocorrência de VAF, a PeNSE perguntou: "Nos últimos 30 dias, você esteve envolvido(a) em alguma briga em que alguma pessoa usou arma de fogo, como revólver ou espingarda?" e para a ocorrência de VOC, a pergunta foi: "Nos últimos 30 dias, você esteve envolvido(a) em alguma briga em que alguma pessoa usou alguma outra arma como faca, canivete, peixeira, pedra, pedaço de pau ou garrafa?". Aqueles que responderam "sim" foram consideradas vítimas.

\section{Análise estatística}

Foram realizadas análises descritivas sobre a prevalência de VFI, VS, VAF e VOC, de acordo com o sexo (masculino e feminino), idade em anos completos $(\leq 13 ; 14 ; 15 ; \geq 16)$, raça/cor autodeclarada (branca; preta; amarela; parda e indígena), coabitação com os pais (nenhum dos pais; apenas a mãe; apenas o pai; com ambos), escolaridade da mãe em anos $(0 ; 1$ a $8 ; 9$ a $11 ; \geq 12)$, tipo de escola (pública ou privada), região do país (Sudeste; Nordeste; Norte; Sul; Centro-Oeste) e consumo de álcool e drogas ilícitas, como maconha, cocaína, crack, cola, loló, lança-perfume, ecstasy, oxy, entre outros (qualquer frequência no último mês anterior à pesquisa).

Todas as análises foram realizadas no programa estatístico STATA (versão 13). A frequência absoluta e relativa das variáveis foi descrita em tabelas. Após, foram realizadas análises bivariadas para testar a associação entre a variável dependente e as variáveis independentes utilizando o teste qui-quadrado utilizando-se o prefixo $s v y$, que estima os pesos amostrais em amostragens complexas, com resultados expressos em prevalências. Foram realizadas análises brutas e ajustadas para potenciais fatores de confusão de acordo com um modelo hierárquico. Neste tipo de análise são levados em consideração um modelo teórico de compreensão da determinação da violência, onde características mais distais estão nos primeiros nível e características mais proximais do desfecho estão no último nível. Nesta abordagem é possível controlar para confusão nas características que compõe o nível e para mediação nas características entre os níveis. As variáveis que atingem valor $\mathrm{p}<0,20$ são mantidas nos níveis subsequentes, caso contrário, são excluídas do modelo. No primeiro nível foram incluídas as características contextuais (macrorregião do país e tipo de escola). No segundo nível foram incluídas as características demográficas (idade e raça/ cor da pele). No terceiro nível as características socioeconômicas (escolaridade materna e coabitação com os pais). No quarto nível as variáveis comportamentais (uso de álcool e drogas ilícitas). Foi adotando o nível de significância de 5\%.

\section{Aspectos éticos}

A pesquisa foi norteada pelo Estatuto da Criança e do Adolescente (Lei no 8.069 de 13 de julho de 1990) e foi aprovada na Comissão Nacional de Ética em Pesquisa (CONEP). 


\section{Resultados}

Foram avaliados 100.540 indivíduos com informações completas para todos os tipos de violência analisados ( $1,5 \%$ com falta de informação). A Tabela 1 apresenta as características da amostra.

Tabela 1. Descrição da amostra, segundo características demográficas, socioeconômicas, comportamentais (PeNSE, 2015; $\mathrm{N}=100.540$ ).

\begin{tabular}{|c|c|}
\hline Variáveis & $\begin{array}{c}\text { Descrição da } \\
\text { amostra } \\
\mathrm{N}(\%)\end{array}$ \\
\hline \multicolumn{2}{|l|}{ Região } \\
\hline Sudeste & $17.539(43,4)$ \\
\hline Norte & $23.568(9,6)$ \\
\hline Nordeste & $35.798(27,7)$ \\
\hline Sul & $9.689(11,8)$ \\
\hline Centro-Oeste & $13.946(7,5)$ \\
\hline \multicolumn{2}{|l|}{ Tipo de escola } \\
\hline Pública & $79.752(85,4)$ \\
\hline Privada & $20.788(14,6)$ \\
\hline \multicolumn{2}{|l|}{ Sexo } \\
\hline Masculino & $48.321(48,4)$ \\
\hline Feminino & $52.219(51,6)$ \\
\hline \multicolumn{2}{|l|}{ Idade } \\
\hline$\leq 13$ & $17.079(18,4)$ \\
\hline 14 & $50.963(51,1)$ \\
\hline 15 & $20.456(19,6)$ \\
\hline$\geq 16$ & $12.042(10,9)$ \\
\hline \multicolumn{2}{|l|}{ Raça/Cor } \\
\hline Branca & $33.338(36,2)$ \\
\hline Preta & $12.610(13,3)$ \\
\hline Parda & $46.238(43,1)$ \\
\hline Amarela & $4.505(4,1)$ \\
\hline Indígena & $3.755(3,3)$ \\
\hline \multicolumn{2}{|c|}{ Escolaridade da mãe (anos) } \\
\hline 0 & $5.405(7,3)$ \\
\hline 1 a 8 & $23.869(35,3)$ \\
\hline 9 a 11 & $23.910(32,9)$ \\
\hline$\geq 12$ & $22.477(24,4)$ \\
\hline \multicolumn{2}{|l|}{ Coabitação com pais } \\
\hline Nenhum dos pais & $6.490(5,7)$ \\
\hline Apenas com a mãe & $31.266(30,6)$ \\
\hline Apenas com o pai & $4.832(4,4)$ \\
\hline Ambos os pais & $57.843(59,4)$ \\
\hline \multicolumn{2}{|l|}{ Consumo de álcool (mês) } \\
\hline Não & $78.235(76,2)$ \\
\hline $\operatorname{Sim}$ & $22.241(23,8)$ \\
\hline \multicolumn{2}{|c|}{ Uso de drogas ilícitas (mês) } \\
\hline Não & $96.687(95,9)$ \\
\hline Sim & $3.829(4,1)$ \\
\hline
\end{tabular}

A maior parte da amostra foi composta por escolares de escolas públicas $(85,4 \%)$, do sexo feminino (51,6\%), com 14 anos de idade $(51,1 \%)$, que autodeclararam sua cor/raça como pardos $(43,1 \%)$, que moravam com ambos os pais $(59,4 \%)$ e cujas mães tinham escolaridade menor ou igual a 8 anos de estudo (42,6\%). Em relação ao uso de substâncias, $23,8 \%$ dos escolares declararam ter consumido álcool e 4,1\% ter feito uso de drogas ilícitas no mês anterior a entrevista.

A Tabela 2 apresenta os resultados da prevalência e fatores associados para toda amostra. Foi observada prevalência de 14,5\%, 7,9\%, 5,7\% e 4,0\% para a vitimização por violência física intrafamiliar (VFI), por objeto perfurocortante (VOC), arma de fogo (VAF) e violência sexual (VS), respectivamente. A VFI foi mais frequente na região Sudeste, a VAF foi mais frequente na região Centro-Oeste e a VOC e VS foram mais frequentes na região Norte do país. A vitimização por VFI e VS foi maior entre as meninas, enquanto a VAF e VOC foi maior entre os meninos. $\mathrm{O}$ autor mais frequente de VS foi o namorado(a) ou ex-namorado(a) da vítima (dados não apresentados na tabela). Todas as formas de vitimização por violência foram mais frequentes em escolares das escolas públicas, entre aqueles com 16 anos ou mais, filhos(as) de mães com nenhuma ou baixa escolaridade e que fizeram uso de álcool ou substâncias ilícitas no mês anterior a entrevista. Observou-se que a vitimização por todos os tipos violência foi maior à medida que aumentava a idade dos escolares. A vitimização por VFI e VS foi mais frequente entre escolares que autorrelataram sua cor da pele/raça como amarela, enquanto a vitimização por VAF e VOC foi mais comum entre aqueles de cor da pele/raça preta. Com exceção da VAF, que foi mais frequente entre os escolares que residiam apenas com o pai, a vitimização por VOC, VFI e VS foi mais frequente entre os escolares que residiam sem nenhum dos pais.

A Tabela 3 apresenta os resultados da análise bruta (razão de prevalência), estratificada por sexo, para VFI, VS, VAF e VOC. Foram observadas associações entre as características demográficas, socioeconômicas e comportamentais em todos tipos de violência avaliados, com exceção da macrorregião do país e tipo de escola para a VFI e macrorregião do país para VS entre os meninos.

A Tabela 4 apresenta os resultados da análise ajustada (razão de prevalência), estratificada por sexo, para VFI, VS, VAF e VOC. Para ambos os sexos, a VFI foi mais frequente na região Sudeste 
Tabela 2. Prevalência de violência física intrafamiliar (VFI), violência sexual (VS), violência por arma de fogo (VAF) e violência por objeto perfurocortante (VOC) segundo características demográficas, socioeconômicas e comportamentais (PeNSE, 2015; $\mathrm{N}=100.540$ ).

\begin{tabular}{|c|c|c|c|c|}
\hline \multirow{3}{*}{ Variáveis } & \multicolumn{4}{|c|}{ Vitimização por violência } \\
\hline & VFI & VS & VAF & VOC \\
\hline & $\mathrm{N}(\%)$ & $\mathbf{N}(\%)$ & $\mathrm{N}(\%)$ & $\mathbf{N}(\%)$ \\
\hline Prevalência geral & $14.167(14,5)$ & $4.064(4,0)$ & $5.480(5,7)$ & $7.988(7,9)$ \\
\hline Região & $\mathrm{p}=0,002$ & $\mathrm{p}=0,002$ & $\mathrm{p}<0,001$ & $\mathrm{p}<0,001$ \\
\hline Norte & $3.426(14,0)$ & $1.267(5,3)$ & $1.489(5,9)$ & $2.364(9,7)$ \\
\hline Nordeste & $4.993(14,2)$ & $1.279(3,9)$ & $1.610(4,8)$ & $2.411(6,9)$ \\
\hline Centro-Oeste & $2.055(14,7)$ & $613(4,4)$ & $1.026(7,6)$ & $1.340(9,6)$ \\
\hline Sudeste & $2.656(15,2)$ & $587(3,7)$ & $936(6,0)$ & $1.270(7,9)$ \\
\hline Sul & $1.185(12,9)$ & $378(4,2)$ & $522(5,5)$ & $733(7,8)$ \\
\hline Tipo de escola & $\mathrm{p}=0,004$ & $\mathrm{p}<0,001$ & $\mathrm{p}<0,001$ & $\mathrm{p}<0,001$ \\
\hline Pública & $11.731(14,8)$ & $3.602(4,4)$ & $4.911(6,1)$ & $6.988(8,4)$ \\
\hline Privada & $2.584(13,0)$ & $522(2,0)$ & $672(3,4)$ & $1.130(5,3)$ \\
\hline Sexo & $\mathrm{p}=0,001$ & $\mathrm{p}=0,009$ & $\mathrm{p}<0,001$ & $\mathrm{p}<0,001$ \\
\hline Masculino & $6.529(13,8)$ & $1.734(3,7)$ & $3.751(7,9)$ & $5.218(10,6)$ \\
\hline Feminino & $7.786(15,1)$ & $2.390(4,3)$ & $1.832(3,7)$ & $2.900(5,4)$ \\
\hline Idade & $\mathrm{p}<0,001$ & $\mathrm{p}<0,001$ & $\mathrm{p}<0,001$ & $\mathrm{p}<0,001$ \\
\hline$\leq 13$ & $2.163(13,8)$ & $428(2,4)$ & $504(3,7)$ & $789(5,0)$ \\
\hline 14 & $6.800(13,5)$ & $1.726(3,3)$ & $2.221(4,6)$ & $3.458(6,8)$ \\
\hline 15 & $3.253(16,2)$ & $1.091(5,6)$ & $1.600(8,2)$ & $2.209(10,5)$ \\
\hline$\geq 16$ & $2.099(17,4)$ & $879(7,3)$ & $1.258(10,2)$ & $1.662(13,4)$ \\
\hline Raça/Cor & $\mathrm{p}<0,001$ & $\mathrm{p}<0,001$ & $<0,001$ & $\mathrm{p}<0,001$ \\
\hline Branca & $4.215(13,1)$ & $1.153(3,3)$ & $1.583(4,9)$ & $2.336(7,0)$ \\
\hline Preta & $2.129(16,8)$ & $632(5,2)$ & $1.032(8,6)$ & $1.355(10,6)$ \\
\hline Parda & $6.597(14,5)$ & $1.900(4,1)$ & $2.446(5,5)$ & $3.693(7,8)$ \\
\hline Amarela & $746(18,1)$ & $228(5,5)$ & $265(5,9)$ & $353(7,8)$ \\
\hline Indígena & $615(16,1)$ & $208(5,1)$ & $252(6,7)$ & $3.784(10,1)$ \\
\hline Escolaridade da mãe (anos) & $\mathrm{p}<0,001$ & $\mathrm{p}<0,001$ & $\mathrm{p}<0,001$ & $\mathrm{p}<0,001$ \\
\hline 0 & $974(19,5)$ & $365(7,7)$ & $491(9,5)$ & $617(11,2)$ \\
\hline 1 a 8 & $3.580(15,1)$ & $1.134(4,3)$ & $1.411(5,9)$ & $2.052(8,4)$ \\
\hline 9 a 11 & $3.343(14,6)$ & $878(3,5)$ & $1.173(4,9)$ & $1.763(7,3)$ \\
\hline$\geq 12$ & $2.985(13,0)$ & $756(3,4)$ & $1.082(5,2)$ & $1.588(7,0)$ \\
\hline Coabitação com pais & $\mathrm{p}<0,001$ & $\mathrm{p}<0,001$ & $\mathrm{p}<0,001$ & $\mathrm{p}<0,001$ \\
\hline Nenhum dos pais & $1.108(17,2)$ & $462(7,1)$ & $519(8,1)$ & $703(11,4)$ \\
\hline Apenas com a mãe & $5.165(16,9)$ & $1.487(4,8)$ & $1.838(6,1)$ & $2.804(8,5)$ \\
\hline Apenas com o pai & $735(15,2)$ & $271(5,8)$ & $365(8,9)$ & $500(10,2)$ \\
\hline Ambos os pais & $7.285(12,9)$ & $1.896(3,2)$ & $2.849(5,1)$ & $4.096(7,1)$ \\
\hline Consumo de álcool (mês) & $\mathrm{p}<0,001$ & $\mathrm{p}<0,001$ & $\mathrm{p}<0,001$ & $\mathrm{p}<0,001$ \\
\hline Não & $8.940(11,4)$ & $2.333(2,8)$ & $2.705(3,6)$ & $4.018(4,8)$ \\
\hline $\operatorname{Sim}$ & $5.357(24,5)$ & $1.788(8,0)$ & $2.862(12,7)$ & $4.082(17,8)$ \\
\hline Uso de drogas ilícitas (mês) & $\mathrm{p}<0,001$ & $\mathrm{p}<0,001$ & $\mathrm{p}<0,001$ & $\mathrm{p}<0,001$ \\
\hline Não & $13.031(13,7)$ & $3.546(3,7)$ & $4.422(4,8)$ & $6.638(6,7)$ \\
\hline Sim & $1.273(33,0)$ & $573(12,4)$ & $1.155(27.8)$ & $1.470(35,4)$ \\
\hline
\end{tabular}

em comparação a região Sul e maior à medida que aumentava a idade. Em relação a raça/cor de pele, observou-se a entre $30-40 \%$ maior prevalência para VFI entre os meninos com raça/cor parda $(\mathrm{RP}=1,37$; IC95\% 1,$10 ; 1,71)$ e entre as meninas que autodeclararam sua cor/raça como preta $(\mathrm{RP}=1,34$; IC95\% 1,18; 1,51), parda ou indígena $(\mathrm{RP}=1,36 \mathrm{IC} 95 \% 1,14 ; 1,62)$. A VFI foi 


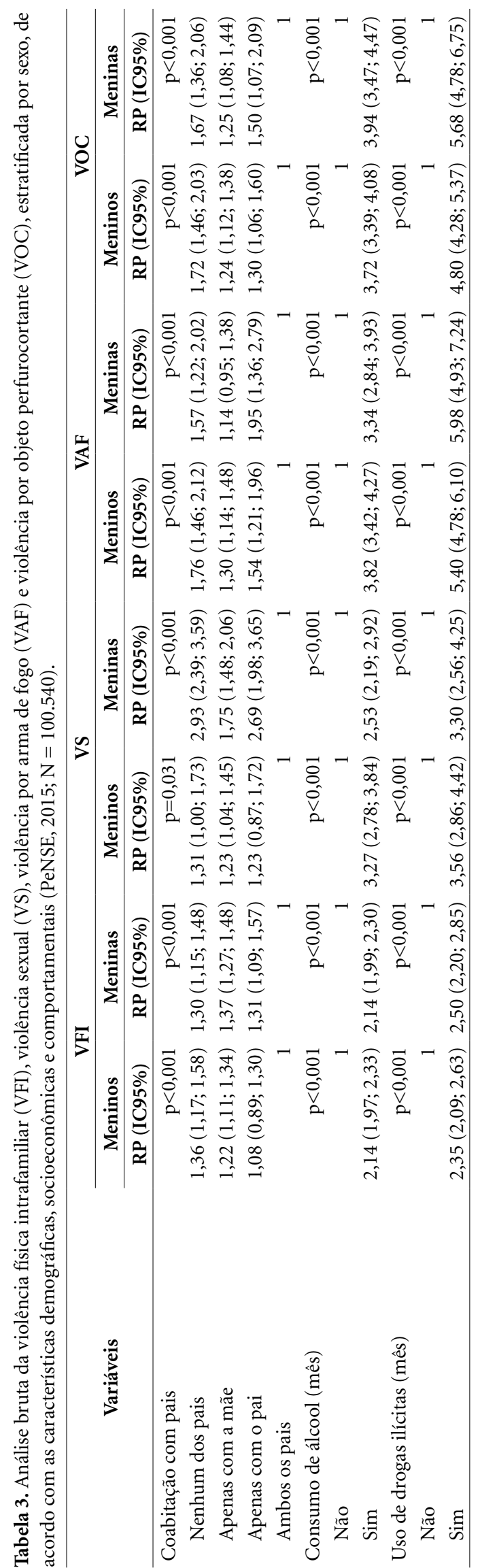

cerca de $40 \%$ mais frequente entre filhos de mães sem escolaridade ( 0 anos de estudo) para ambos os sexos em comparação as mais com maior escolaridade. As meninas das escolas públicas relataram maior ocorrência da VFI em comparação às escolas privadas, associação não observada entre os meninos. Os meninos que não moravam com nenhum dos pais apresentaram maior frequência de VFI enquanto as meninas que moravam apenas com a mãe ou apenas com o pai relataram maior ocorrência de VFI em comparação aqueles/as que moravam com ambos os pais. Os escolares que consumiram álcool no último mês relataram duas vezes mais a ocorrência de VFI em comparação aqueles que não relataram o uso. A VFI foi mais frequente naqueles que relataram uso de drogas ilícitas no último mês, tanto em meninos ( $\mathrm{RP}=1,40$; IC95\% 1,21; 1,62) como em meninas $(\mathrm{RP}=1,61$; IC95\% 1,$35 ; 1,92)$.

$\mathrm{O}$ relato da VS entre as meninas foi menos frequente na região Sudeste $(\mathrm{RP}=0,76$; IC95\% $0,61 ; 0,95)$ e Nordeste ( $\mathrm{RP}=0,79$; IC95\% 0,66; $0,96)$ em comparação a região Sul. Não houve diferença significativa para VS de acordo com a região do país entre os meninos. A vitimização por VS em escolares foi cerca de duas vezes maior entre os meninos e 2,5 maior entre as meninas das escolas públicas em comparação as escolas privadas. Em ambos os sexos se observou maior vitimização por VS à medida que aumentava a idade do respondente. Meninas da cor/raça preta e parda relataram cerca de $40 \%$ e $70 \%$ mais vitimização por VS em comparação as meninas da cor/raça branca, não sendo observada associação da cor/raça com VS entre os meninos. O maior nível de escolaridade materna foi um fator de proteção para a vitimização de VS entre os meninos, mas não entre as meninas. As meninas que moravam apenas com as mães relataram as menores frequências de VS, diferença não observada entre os meninos. A VS foi maior entre os escolares que relataram o uso de álcool e drogas ilícitas, sendo maior entre os meninos do que entre as meninas.

A VAF foi maior na região Centro-Oeste em ambos os sexos em comparação a região Sul e cerca de duas vezes maior em alunos de escolas públicas em comparação com as escolas privadas. Também foi observado que a vitimização por VAF aumentava de acordo com a idade do respondente. Meninos (RP = 1,43; IC95\% 1,22; $1,67)$ e meninas $(\mathrm{RP}=1,69 ; \mathrm{IC} 95 \% 1,30 ; 2,18)$ que autodeclararam sua cor/raça como preta apresentaram as maiores frequências de vitimização por arma de fogo em comparação com 


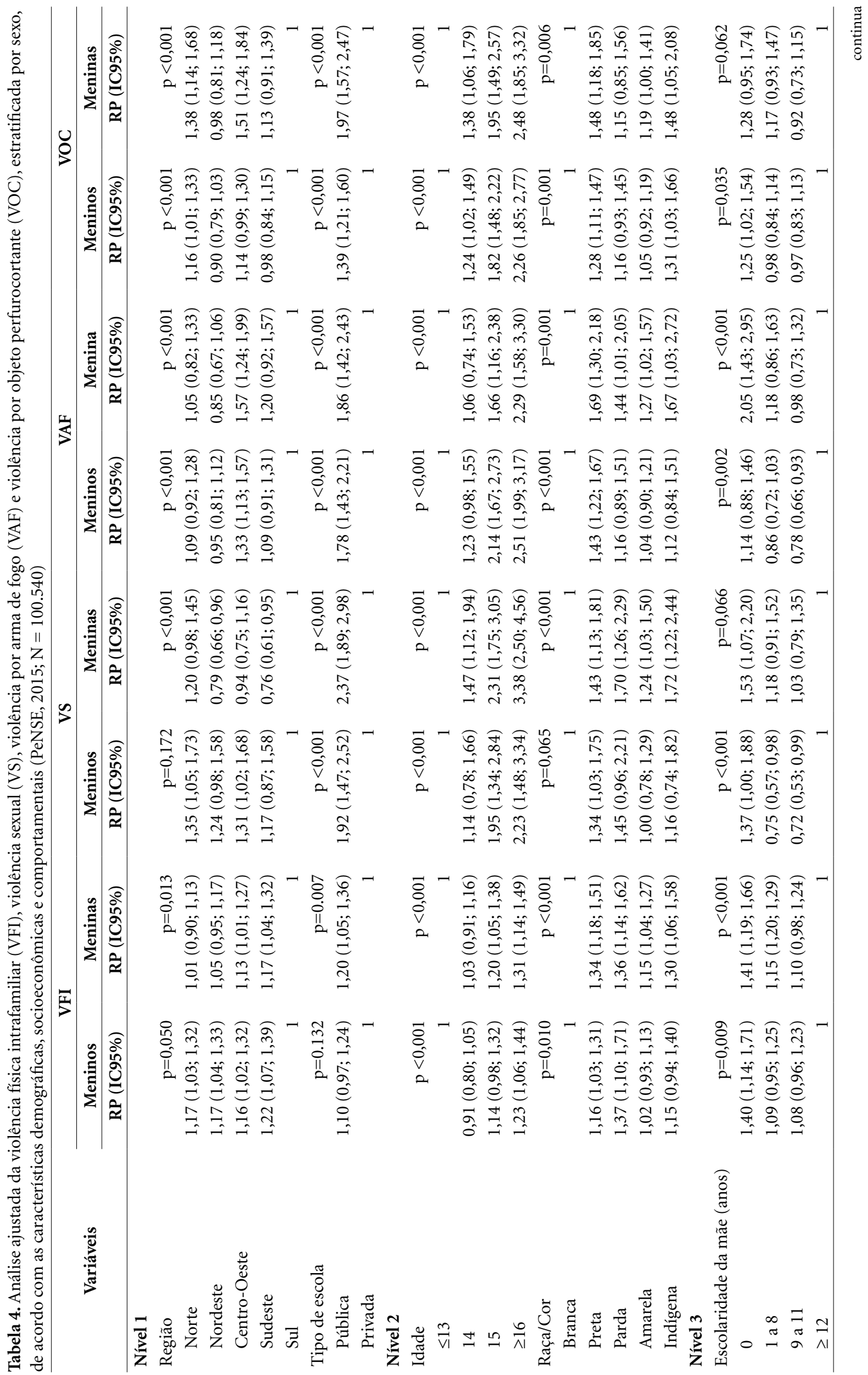




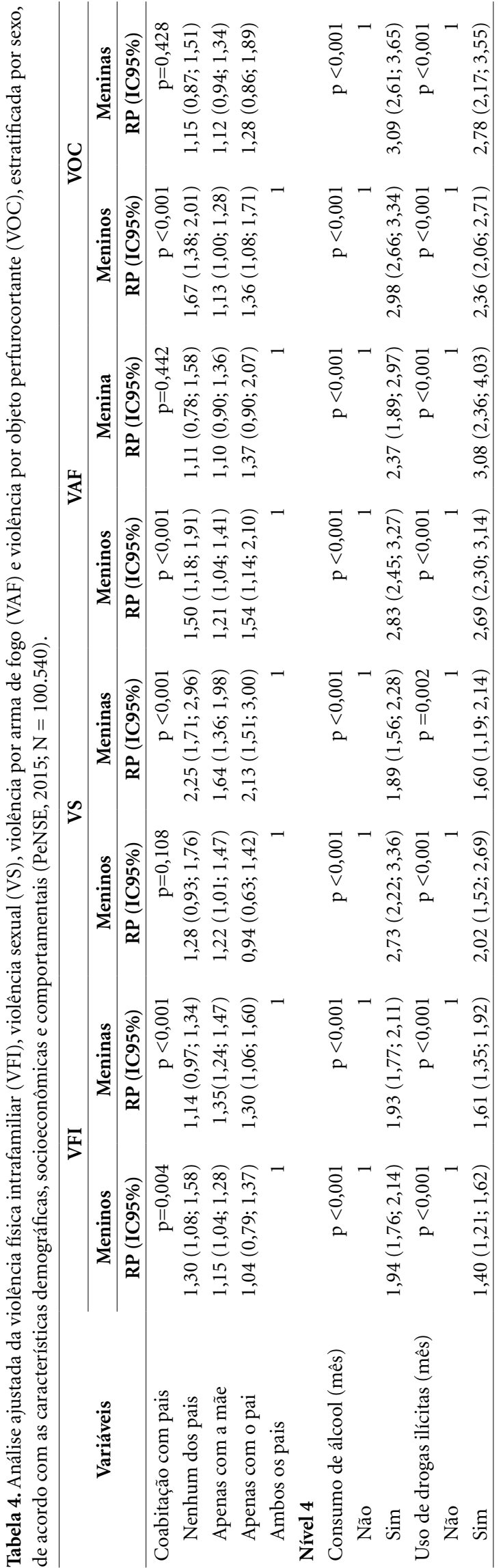

aqueles da cor/raça branca. A ocorrência de VAF tende a diminuir à medida que aumentava a escolaridade materna em ambos os sexos. Meninos filhos de mães com escolaridade maior ou igual a 12 anos apresentaram uma redução de $66 \%$ a $93 \%$ na vitimização por arma de fogo $(\mathrm{RP}=$ 0,78; IC95\% 0,66; 0,93). A VAF foi cerca de três vezes maior entre os meninos e cerca de 2,4 vezes maior entre as meninas que consumiram álcool no mês anterior a entrevista. As meninas que relataram uso de drogas ilícitas apresentaram três vezes mais chance de VAF do que aquelas que não relataram uso de drogas no mês anterior, padrão observado também entre os meninos.

A VOC foi mais frequente entre os meninos da região Norte $(\mathrm{RP}=1,16$; IC95\% 1,01; 1,33) e meninas da região Centro-Oeste $(\mathrm{RP}=1,51$; IC95\% 1,24; 1,84). As meninas de escolas públicas apresentaram duas vezes mais probabilidade de serem vítimas por VOC do que alunos de escolas privadas, enquanto que nos meninos esta probabilidade foi $40 \%$ maior do que aqueles de escolas privadas. Escolares da cor/raça preta $\mathrm{e}$ indígena apresentaram as maiores prevalências de VOC em comparação aos estudantes da cor/ raça branca, sendo ainda maior entre as meninas do que entre os meninos. Foi observado que o aumento da escolaridade materna estava associado a diminuição da VOC entre os meninos. O mesmo padrão foi observado entre as meninas, no entanto, esta diferença não foi estatisticamente significativa. Meninos que moravam sem nenhum dos pais apresentaram cerca de $70 \%$ maior probabilidade do envolvimento em VOC em comparação aqueles que moravam com ambos os pais. Escolares de ambos os sexos que relataram o uso de álcool e drogas ilícitas apresentaram entre 2 e 3 vezes maior prevalência de VOC em comparação aqueles que não relataram o uso destas substâncias.

\section{Discussão}

Os resultados da PeNSE 2015 evidenciaram maior prevalência de VFI e VS entre as meninas e de VAF e VOC entre os meninos. A literatura apontou que, sendo a VFI e a VS agressões que ocorrem dentro das relações familiares e afetivas, estas tendem a contribuir para o desenvolvimento de problemas de regulação emocional e comportamental que prejudicariam a estabilidade das relações interpessoais das vítimas a longo prazo ${ }^{12,16}$. Por outro lado, em outros estudos, a VAF e VOC estiveram associadas com a vitimização por vio- 
lência no passado, exposição a comportamentos agressivos dos pares ${ }^{17} \mathrm{e}$ fazer uso de drogas ilícitas foram fatores de risco associados ${ }^{18}$. Tais fatores de risco podem viabilizar uma dessensibilização emocional frente a situações violentas e posterior desenvolvimento dos comportamentos externalizantes, como a impulsividade - preditor de envolvimento em brigas com armas - similar ao encontrado em outros estudos. Ainda, dentre as quatro violências analisadas, a VFI foi a mais prevalente. Esse achado também foi encontrado em uma revisão sistemática que reuniu 43 estudos sobre violência e apontou a alta prevalência de abusos físicos nessa população, majoritariamente perpetrados pelos familiares das vítimas ${ }^{19}$. Em relação a VS, esse estudo identificou os parceiros íntimos como principais autores do abuso. Considera-se ainda que há uma tendência de subnotificação dessa violência, quando perpetrada por parceiro íntimo, uma vez que tem menos chances de ser reconhecida pelas vítimas como abuso ${ }^{20}$. Nota-se então que, de forma geral, as meninas sofreram mais violência dentro de relações familiares e afetivas. Esse perfil da violência pode contribuir para o desenvolvimento de problemas de regulação emocional e comportamental, como reatividade, ruminações e impulsividade, que prejudicariam à estabilidade das relações interpessoais das vítimas ${ }^{21}$.

Por outro lado, os meninos apresentaram maior frequência de envolvimento em VAF e VOC. A maior prevalência do sexo masculino nessa violência também foi encontrada em estudos de base escolar na França ${ }^{17}$ e Tailândia ${ }^{18}$ com escolares de 11 a 19 anos. Esses estudos destacaram que vitimização por violência no passado, comportamentos agressivos dos pares $^{17}$ e fazer uso de drogas ilícitas foram fatores de risco associados $^{18}$. No Brasil, após uma análise do perfil de vítimas de violência armada, os principais comportamentos de risco apontados foram o consumo de drogas ilícitas e vinculação ao tráfi$\mathrm{CO}^{22}$. A literatura apontou que o desenvolvimento dos comportamentos de risco pode derivar da exposição a múltiplos contextos de violência no passado, viabilizando uma dessensibilização emocional frente a situações violentas e posterior desenvolvimento dos comportamentos externalizantes, como a impulsividade - preditor de envolvimento em brigas com armas ${ }^{16}$.

A VFI e VS também foi mais frequente entre a população que declarou sua cor de pele/raça como amarela. Já a VAF e VOC foram mais frequentes entre aqueles que declararam sua cor de pele como preta. No primeiro caso, não foram en- contrados estudos que indicassem e explicassem a alta prevalência de violência contra essa população no Brasil ${ }^{23,24}$. Já no segundo caso, sabendo que o uso de armas em brigas está associado ao aumento de riscos de lesões graves ou morte ${ }^{1}$, o Índice de Vulnerabilidade Juvenil à Violência 2017 discutiu a prevalência de violência armada seguida de homicídio entre os adolescentes. Os resultados indicaram que os jovens negros têm 2,7 vezes mais chances de sofrer desse tipo de violência do que os jovens de cor de pele branca ${ }^{25}$. Esse cenário aponta uma desigualdade racial em relação à vitimização por violência armada no Brasil, que pode refletir as desvantagens materiais e sociais às quais essa população está exposta ${ }^{14}$.

As quatro formas de violência analisadas foram mais frequentes de acordo com o aumento da idade dos respondentes, similar ao apontado pelo inquérito em serviços de urgência no Brasil em 2014 em relação a $\mathrm{VFI}^{6}$, também nos estudos de Devries et al. ${ }^{19}$ em relação à VS por parceiros íntimos e de Bègue et al. ${ }^{17}$ quanto à violência armada. A explicação, pelo modelo de compreensão ecológico, é que a ocorrência da violência engloba uma série de fatores de risco, incluindo características dos adolescentes, dos cuidadores, da comunidade local e os aspectos sociais, econômicos e culturais ${ }^{13}$. Por exemplo, entre os fatores de risco na família, se pode apontar conflitos e vulnerabilidades como o abuso de álcool e drogas ilícitas ${ }^{18}$, sobrecarga do cuidador e crenças de disciplina e correção por agressão ${ }^{26}$. Por outro lado, em relação às características dos adolescentes, a própria idade sinaliza um período de adoção de novos comportamentos e autonomia que podem viabilizar a exposição ao risco fora da supervisão de adultos ${ }^{27}$. Ainda, cabe ressaltar que os adolescentes com 16 anos ou mais não estão na faixa etária esperada para o $9^{\circ}$ ano do ensino fundamental, indicando um possível atraso escolar da população de risco desse estudo. Na literatura, se evidenciou a associação significativa entre a reprovação escolar e a vitimização de violência ${ }^{17,27}$.

Em relação ao consumo de álcool e drogas ilícitas, embora o recorte desse estudo admita um viés de causalidade reversa, a literatura indica uma bidirecionalidade nessa associação, sendo que tanto as substâncias psicoativas podem preceder a violência, como a violência pode preceder o uso dessas substâncias ${ }^{28,29}$. A explicação para o primeiro caso é que o álcool e as drogas agem diretamente sob o funcionamento cognitivo do indivíduo, dificultando o controle de impulsos, a percepção de risco e o planejamento estratégico para sair de situações perigosas ${ }^{29-31}$. Para o segun- 
do caso, as vítimas de violência podem consumir e buscar as substâncias como uma estratégia de enfrentamento, ainda que ineficaz, para modulação emocional ${ }^{32-34}$.

Esse estudo também encontrou maior frequência de violência entre os filhos de mães sem escolaridade e entre os estudantes de escolas públicas, como evidenciado nas edições anteriores da PeNSE ${ }^{35,36}$. Esse achado pode refletir uma vulnerabilidade social ao ponto que a baixa escolaridade pode acarretar dificuldades de estabilidade no mercado de trabalho e consequentemente em baixa renda familiar. Em virtude disso, os cuidadores também podem ficar mais tempo fora de casa e/ou em subempregos, o que prejudicaria a supervisão desses adolescentes ${ }^{37}$. Ainda sobre a estrutura familiar, nesse estudo a violência foi mais frequente entre aqueles que não coabitavam com ambos os pais , como similar ao encontrado em um estudo de base escolar com 8.494 alunos de até 18 anos, na Suécia ${ }^{38}$. Esse cenário o que aponta uma provável rede familiar de risco, de pouco suporte, onde a inserção do adolescente viabiliza a vitimização por violência ${ }^{39}$.

Sobre a ocorrência da violência nas macrorregiões do Brasil, nesse estudo a VFI foi mais frequente na região Sudeste e a VAF e VOC, foram mais frequentes no Centro-Oeste e no Norte, respectivamente, similar ao encontrado na edição anterior da PeNSE, em $2012^{36}$. A VS também foi mais frequente na região Norte do país, como apontou um estudo que comparou a prevalência de VS em quatro capitais brasileiras e destacou Belém com o maior número de $\operatorname{casos}^{40}$.

Os fatores associados à violência, apontados nesse estudo, são importantes indicadores a serem considerados no planejamento de ações interventivas para redução da violência e/ou de suas consequências. Entretanto, sabe-se ainda que além dos fatores de risco comuns, existem fatores de proteção que podem minimizar as ocorrências da violência e seus danos. Um exemplo de programa de prevenção à violência interpessoal que focou em fatores de proteção foi o Fast Track, nos Estados Unidos. O foco se deu no desenvolvimento de habilidades sociais, regulação emocional e resolução de problemas com indivíduos de 6 a 11 anos e, após 15 anos de acompanhamento, se observou a eficácia do programa na redução de comportamentos de risco e problemas de conduta, associados a ocorrência de violência armada ${ }^{41}$. A eficácia da intervenção também foi devido a sua ampla abordagem, uma vez que o programa envolveu o público alvo, os pais e/ou responsáveis, bem como os professores e funcionários das escolas ${ }^{41}$.
No Brasil, estudos sobre intervenções à violência tiveram maior foco na capacitação de professores e profissionais da saúde por meio da formação inicial e continuada. Para os professores pois, em decorrência da alta frequência de casos descobertos na escola, muitos não se sentem preparados para lidar com a violência as quais seus alunos podem estar submetidos ${ }^{42}$. Ainda que a escola notifique os casos aos órgãos competentes, essa não é a única possibilidade de enfrentamento à violência. A capacitação se faz importante para estimular a discussão sobre o fenômeno e a participação na elaboração e efetivação de intervenções e políticas públicas ${ }^{42}$. Ain$\mathrm{da}$, as ações devem estar articuladas com outras áreas comprometidas com a garantia dos direitos das crianças e adolescentes, como a Saúde. Entretanto, a literatura apontou que, para intervir nos casos de violência, os serviços de saúde encontram desafios referentes à utilização dos sistemas de notificação, bem como em relação à rede de encaminhamentos das vítimas às redes de saúde mental ${ }^{10}$. Assim, se faz necessário o investimento na capacitação da equipe de saúde e da educação.

Por fim, como vantagens da PeNSE, se destaca sua importância como a mais ampla pesquisa de base escolar com adolescentes no Brasil, por sua abrangência nacional e a representatividade da amostra. Soma-se isso ao fato de que pesquisas sobre violência tendem a utilizar registros e dados secundários provenientes de serviços ou sistemas de saúde, sustentando um viés de subnotificação nos resultados. Além disso, visto que os casos de violência podem ser muitas vezes omitidos ou negados, por medo e/ou vergonha, a PeNSE elaborou a pesquisa a partir do questionário autoaplicado, proporcionando o sigilo das informações. Já as limitações desse estudo estão relacionadas ao instrumento utilizado para mensurar a violência no contexto escolar, uma vez que a coleta de informações foi pelo autorrelato, a partir de um inquérito extenso, não possibilitando a utilização de escalas próprias para mensuração da violência. Ressalta-se também que a PeNSE não alcança os alunos com evasão escolar e/ou absenteísmo, que são fatores associados a situações de violência ${ }^{43}$.

\section{Considerações finais}

A PeNSE vem dando visibilidade a diversas condições às quais os adolescentes estão expostos, inclusive a violência, que é um problema de saúde pública que agrava a vulnerabilidade dos adoles- 
centes brasileiros. Os resultados de 2015 indicaram maior prevalência de violência armada entre os meninos e de violência física intrafamiliar e violência sexual entre as meninas, principalmente entre os adolescentes mais velhos, alunos de escolas públicas, filhos de mães com baixa escolaridade, que moravam sem os pais e que fizeram uso de álcool ou substâncias ilícitas.

Os achados vêm ressaltar a importância das políticas públicas de proteção social e incentivar a elaboração de estratégias de intervenção para a violência contra os adolescentes. Essas estratégias podem ser elaboradas para trabalhar diretamente com os adolescentes, especialmente no ambiente escolar, visto que esse ambiente possa ser propício para intervenções, uma vez que pode alcançar mais indivíduos em um determinado período, bem como podem incluir capacitações durante as formações iniciais e continuadas das equipes de saúde e educação.

\section{Colaboradores}

TN Munhoz e FBP Terribele participaram da concepção do estudo, análise e interpretação dos dados, interpretação dos resultados e escrita de todas as versões do manuscrito. Todos os autores revisaram criticamente e aprovaram a versão final. 


\section{Referências}

1. World Health Organization (WHO). Global status report on violence prevention. Genebra: WHO; 2014.

2. Azevedo MA, Guerra VA. Um cenário em (des)construção. In: UNICEF, organizador. Direitos negados - A violência contra crianças e adolescentes no Brasil. Brasília: UNICEF; 2006. p. 14-27.

3. Carlson JS, Yohannan J, Darr CL, Turley MR, Larez NA, Perfect MM. Prevalence of adverse childhood experiences in school-aged youth: A systematic review (1990-2015). Inter Jour Sch Educ Psych 2019; 1-22.

4. Hillis S, Mercy J, Amobi A, Kress H. Global Prevalence of Past-year Violence Against Children: A Systematic Review and Minimum Estimates. Pediatr 2016; 137(3):e20154079.

5. Rodrigues NCP, O’Dwyer G, Andrade MKN, Flynn MB, Monteiro DLM, Lino VTS. The increase in domestic violence in Brazil from 2009-2014. Cien Saude Colet 2017; 22(9):2873-2880.

6. Malta DC, Bernal RTI, Pugedo FSF, Lima CM, Mascarenhas MDM, Jorge AO, Melo EM. Violências contra adolescentes nas capitais brasileiras, segundo inquérito em serviços de urgência. Cien Saude Colet 2017; 22(9):2899-2908.

7. Santos MJ, Mascarenhas MDM, Rodrigues MTP, Monteiro RA. Caracterização da violência sexual contra crianças e adolescentes na escola-Brasil, 20102014. Epidemiol Serv Saúde 2018; 27:e2017059.

8. Malta DC, Souza ER, Silva MMA, Silva CS, Andreazzi MAR, Crespo C, Mascarenhas MDM, Porto DL, Figueroa ALG, Morais Neto OL. Vivência de violência entre escolares brasileiros: resultados da Pesquisa Nacional de Saúde do Escolar (PeNSE). Cien Saude Colet 2010; 15(Supl. 2):3053-3063.

9. Lemos FCS. O Estatuto da Criança e do Adolescente no Brasil atual. Rev Psic Pol 2008; 8(15):93-106.

10. Von Hohendorff J, Habigzang LF, Koller SH. Psicoterapia para crianças e adolescentes vítimas de violência sexual no sistema público: panorama e alternativas de atendimento. Psicol Cien Prof 2015; 35(1):182-198.

11. Garbin CAS, Arcieri RM, Araújo PC, Garbin AJÍ. Identificação dos casos de violência contra crianças em escolas municipais de ensino básico de Araçatuba, São Paulo. Em Extensão 2017; 15(2):94-108.

12. Norman RE, Byambaa M, De R, Butchart A, Scott J, Vos $\mathrm{T}$. The long-term health consequences of child physical abuse, emotional abuse, and neglect: a systematic review and meta-analysis. PLoS Medicine 2012; 9(11):e1001349.

13. Cicchetti D, Valentino K. An ecological-transactional perspective on child maltreatment: Failure of the average expectable environment and its influence on child development. In: Developmental psychopathology: Risk, disorder, and adaptation. Nova Jersey: John Wiley \& Sons Inc; 2015. p. 129-201.

14. Minayo MCS. Violência e saúde. Rio de Janeiro: Fiocruz; 2006.

15. Instituto Brasileiro de Geografia e Estatísticas (IBGE). Pesquisa Nacional de Saúde do Escolar, 2009. Rio de Janeiro: IBGE; 2009.

16. Mrug S, Madan A, Windle M. Emotional Desensitization to Violence Contributes to Adolescents' Violent Behavior. J Abnor Psych 2016; 44(1):75-86.
17. Bègue L, Roché S, Duke AA. Young and armed: a cros$\mathrm{s}$-sectional study on weapon carrying among adolescents. Psych, Crime Law 2016; 22(5):455-472.

18. Saiphoklang O-a, Wongboonsin K, Wongboonsin P, Perngparn U, Cottler LB. The association between weapon carrying and health risk behaviors among adolescent students in Bangkok, Thailand. J Interp Viol 2017; 32(20):3111-3130.

19. Devries K, Knight L, Petzold M, Merrill KG, Maxwell L, Williams A, Cappa C, Chan KL, Garcia-Moreno C, Hollis N, Kress H, Peterman A, Walsh SD, Kishor S, Guedes A, Bott S, Butron Riveros BC, Watts C, Abrahams $\mathrm{N}$. Who perpetrates violence against children? A systematic analysis of age-specific and sex-specific data. BMJ Paed Open 2018; 2(1):e000180.

20. Jaffe AE, Steel AL, DiLillo D, Messman-Moore TL, Gratz KL. Characterizing Sexual Violence in Intimate Relationships: An Examination of Blame Attributions and Rape Acknowledgment. J Interp Viol 2017; 886260517726972.

21. Heleniak C, Jenness JL, Stoep AV, McCauley E, McLaughlin KA. Childhood Maltreatment Exposure and Disruptions in Emotion Regulation: A Transdiagnostic Pathway to Adolescent Internalizing and Externalizing Psychopathology. Cog Ther Reserch 2016; 40(3):394-415.

22. Maciel PR, Souza MR, Rosso CFW. Estudo descritivo do perfil das vítimas com ferimentos por projéteis de arma de fogo e dos custos assistenciais em um hospital da Rede Viva Sentinela. Epidemiol Serv Saúde 2016; 25:607-616.

23. Delziovo CR, Bolsoni CC, Nazário NO, Coelho EBS Características dos casos de violência sexual contra mulheres adolescentes e adultas notificados pelos serviços públicos de saúde em Santa Catarina, Brasil. Cad Saúde Publica 2017; 33:1-13.

24. Avanci JQ, Pinto LW, Assis SG. Atendimento dos casos de violência em serviços de urgência e emergência brasileiros com foco nas relações intrafamiliares e nos ciclos de vida. Cien Saude Colet 2017; 22(9):28252840.

25. Brasil. Índice de vulnerabilidade juvenil à violência 2017: desigualdade racial, municípios com mais de 100 mil habitantes. São Paulo: Fórum Brasileiro de Segurança Pública; 2017.

26. Pires AL, Miyazaki M. Maus-tratos contra crianças e adolescentes: revisão da literatura para profissionais da saúde. Arq Cien Saude 2005; 12(1):42-49.

27. Patias ND, Dell'Aglio DD. Prevalencia de exposición a violencia directa e indirecta: un estudio con adolescentes de colegios públicos. Act Colom Psicol 2017; 20(1):101-111.

28. East PL, Hokoda A. Risk and protective factors for sexual and dating violence victimization: a longitudinal, prospective study of Latino and African American adolescents. J Youth Adolesc 2015; 44(6):1288-300.

29. Shorey RC, Moore TM, McNulty JK, Stuart GL. Do alcohol and marijuana increase the risk for female dating violence victimization? A prospective daily diary investigation. Psych Viol 2016; 6(4):509.

30. Spear LP. Effects of adolescent alcohol consumption on the brain and behaviour. Nature Rev Neuroscience 2018; 19(4):197. 
31. Fontes MA, Bolla KI, Cunha PJ, Almeida PP, Jungerman F, Laranjeira RR, Bressan RA, Lacerda AL. Cannabis use before age 15 and subsequent executive functioning. Brit J Psych 2011; 198(6):442-447.

32. Goldstein AL, Flett GL, Wekerle C. Child maltreatment, alcohol use and drinking consequences among male and female college students: An examination of drinking motives as mediators. Add Behav 2010; 35(6):636-639.

33. Jester JM, Steinberg DB, Heitzeg MM, Zucker RA. Coping expectancies, not enhancement expectancies, mediate trauma experience effects on problem alcohol use: A prospective study from early childhood to adolescence. J Stud Alcohol Drugs 2015; 76(5):781789 .

34. Miranda Jr R, Meyerson LA, Long PJ, Marx BP, Simpson SM. Sexual assault and alcohol use: Exploring the self-medication hypothesis. Viol Vict 2002; 17(2):205.

35. Andrade SSCA, Yokota RTC, Sá NNB, Silva MMA, Araújo WN, Mascarenhas MDM, Malta DC. Relação entre violência física, consumo de álcool e outras drogas e bullying entre adolescentes escolares brasileiros. Cad Saúde Publica 2012; 28:1725-1736.

36. Malta D, Mascarenhas M, Dias A, Prado R, Lima C, Silva $\mathrm{M}$, Silva Júnior J. Situações de violência vivenciadas por estudantes nas capitais brasileiras e no Distrito Federal: resultados da Pesquisa Nacional de Saúde do Escolar (PeNSE 2012). Rev Bras Epidemiol 2014; 17:158-171.

37. Cardia N, Schiffer S. Violência e desigualdade social. Cien Cultura 2002; 54(1):25-31.

38. Annerbäck EM, Wingren G, Svedin CG, Gustafsson PA. Prevalence and characteristics of child physical abuse in Sweden-findings from a population-based youth survey. Acta Paediatrica 2010; 99(8):1229-1236.

39. Gomide PIC, Salvo CG, Pinheiro DPN, Sabbag GM. Correlação entre práticas educativas, depressão, estresse e habilidades sociais. PsicoUSF 2005; 10(2):169178.
40. Deslandes SF, Vieira LJES, Cavalcanti LF, Silva RM. Atendimento à saúde de crianças e adolescentes em situação de violência sexual, em quatro capitais brasileiras. Interface (Botucatu) 2016; 20(59):865-877.

41. Sorensen LC, Dodge KA, Group CPPR. How does the fast track intervention prevent adverse outcomes in young adulthood? Child Development 2016; 87(2):429-445.

42. Pereira ABM, Conceição MIG, Borges LFF. Reflexões sobre formação de professores para o enfrentamento da violência intrafamiliar contra crianças. Tecnia 2018; 2(2):63-83.

43. Morrow AS, Villodas MT. Direct and Indirect Pathways From Adverse Childhood Experiences to High School Dropout Among High Risk Adolescents. J Reser Adoles 2018; 28(2):327-341.

Artigo apresentado em 21/09/2018

Aprovado em 15/04/2019

Versão final apresentada em 17/04/2019

Editores chefes: Romeu Gomes, Antônio Augusto Moura da Silva 